\title{
La littérature grecque contemporaine traduite en turc : un capital de sympathie
}

Greek Contemporary Literature Translated into Turkish: A Wave of Sympathy

Türkçe'ye Çevrilen Çağdaş Yunan Edebiyatı : Sempati Sermayesi

\section{Faruk Bilici}

\section{OpenEdition}

\section{Journals}

Édition électronique

URL : https://journals.openedition.org/ceb/3198

DOI : $10.4000 /$ ceb.3198

ISSN : 2261-4184

Éditeur

INALCO

Édition imprimée

Date de publication : 1 janvier 2004

ISSN : 0290-7402

Référence électronique

Faruk Bilici, «La littérature grecque contemporaine traduite en turc : un capital de sympathie », Cahiers balkaniques [En ligne], 33 | 2004, mis en ligne le 08 août 2012, consulté le 06 juillet 2021. URL : http:// journals.openedition.org/ceb/3198; DOI : https://doi.org/10.4000/ceb.3198

Ce document a été généré automatiquement le 6 juillet 2021

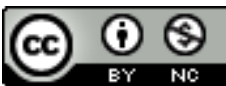

Cahiers balkaniques est mis à disposition selon les termes de la Licence Creative Commons Attribution - Pas d'Utilisation Commerciale 4.0 International. 


\title{
La littérature grecque contemporaine traduite en turc : un capital de sympathie
}

\author{
Greek Contemporary Literature Translated into Turkish: A Wave of Sympathy \\ Türkçe’ye Çevrilen Çağdaş Yunan Edebiyatı : Sempati Sermayesi
}

\section{Faruk Bilici}

1 Si les Turcs disposaient dans la période classique de l'Empire ottoman d'une chancellerie grecque, celle-ci a servi à la correspondance avec l'Empire byzantin et autres puissances occidentales plutôt qu'à une initiation à la littérature ou à d'autres textes. Mehmed II qui, dit-on, comprenait le grec, a fait traduire certains textes grecs en turc ottoman; jusqu'au XIX ${ }^{e}$ siècle, le corps d'interprètes officiels dans la cour ottomane était composé essentiellement de Grecs, et la communauté non-musulmane la plus importante était aussi la grecque. Tous ces facteurs favorables auraient dû susciter un certain intérêt chez les Ottomans pour la langue et surtout pour la production littéraire grecques. Or, jusqu'au XVIII ${ }^{e}$ siècle, le peu de choses que les Ottomans connaissaient de la Grèce ancienne était dû aux Arabes ou aux quelques traductions dans des langues occidentales. Il faut attendre la fameuse période dite des Tulipes (1703-1730) où un mouvement intellectuel est né avec la création de l'imprimerie, pour voir un début de prise de conscience, grâce à Ibrahim Müteferrika. Ainsi, le grand vizir Ibrahim Pacha de Nevşehir (décédé en 1730) créa-t-il sous la présidence du poète Nedim une commission, afin de traduire les classiques de l'Islam, mais aussi, et pour la première fois, la Physica d'Aristote directement du grec ${ }^{1}$.

$2 \mathrm{Au} \mathrm{XIX}^{\mathrm{e}}$ et au début du XX $\mathrm{XX}^{\mathrm{e}}$ siècle, entre 1845 et 1918, on a pu traduire une partie des œuvres d'Hérodote, Xénophon, Ésope, Euripide, Homère, Plutarque, Théocrite, Lucien². Le Bureau de traduction (Tercüme Odasi), créé en 1832, était tourné vers le français et la diplomatie, en conséquence les œuvres littéraires n'ont pas bénéficié des efforts des pouvoirs publics dans ce domaine. Nombreuses œuvres sont traduites à l'initiative privée à partir des différentes langues occidentales y compris du russe. 
3 Mais dans cette étude, il s'agira surtout d'un survol rapide des différents canaux par lesquels la littérature grecque contemporaine a pu entrer dans la vie intellectuelle de la Turquie républicaine.

\section{Varlık: outil littéraire du rapprochement turco-grec}

4 Comme dans beaucoup de domaines, la revue littéraire Varlk a joué un rôle précurseur dans la traduction et la diffusion de la littérature grecque contemporaine en Turquie. Volontariste pour participer à l'œuvre révolutionnaire des Kémalistes, cette revue qui commence à paraître le 15 juillet 1933 (et continue à paraître aujourd'hui), se pose d'emblée comme le promoteur de l'écriture de qualité produite par l'intelligentsia turque. Elle se propose également d'ouvrir ses pages, «pas seulement à la littérature française, comme on le faisait jusqu'à maintenant", mais également à des chefs d'œuvres de l'Occident et à des mouvements littéraires occidentaux ${ }^{3}$. Aussi, la revue publie-t-elle des nouvelles, des extraits de pièces de théâtre, de romans, des poésies traduits des langues occidentales (allemand, français, anglais, espagnol, sans oublier le russe). Son originalité réside essentiellement dans son effort pour initier le lecteur turc à des littératures moins connues, moins diffusées, celles des "voisins ». Aussi, dès le départ et dans le contexte des réformes républicaines, de l'ouverture culturelle et de la modernisation du pays, des traductions de nouvelles du hongrois ${ }^{4}$, du bulgare ${ }^{5}$, mais aussi et surtout du grec ont vu le jour dans cette revue. Contrairement aux autres littératures "régionales » du sud (comme on pouvait s'y attendre, les littératures iranienne, arabe ou géorgienne sont inexistantes dans cette revue, comme d'ailleurs les autres littératures turques du Caucase ou de l'Asie centrale), la littérature grecque des années vingt trouvera régulièrement un écho. Aussi, le premier texte publié dans le numéro 9 (15 novembre 1933) est-il l'extrait du roman roman Vie au tombeau de Stratis Myrivilis. D'autres vont le suivre. Mais il faut noter que durant trois ans, les auteurs étrangers ne sont pas annoncés sur la couverture, seul le nom du traducteur étant

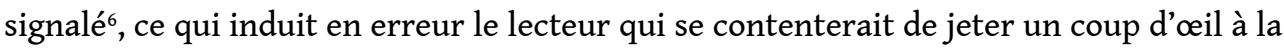
couverture ou aux index.

5 Une recherche systématique dans cette revue entre 1933-1940 donne le tableau quantitatif suivant ${ }^{7}$ :

Tableau 1 : la revue Varlık, textes littéraires traduits du grec (1933-1940) par A. Papazoğlu

\begin{tabular}{|l|l|l|l|l|}
\hline Auteur & Titre & Genre & $\mathrm{n}^{\mathrm{o}}$ & Date \\
\hline Stratis Myrivilis & $\begin{array}{l}\text { Asker Analar } \\
\text { (Les mères militaires) }\end{array}$ & $\mathrm{R}$ & 9 & $\begin{array}{l}15 \text { nov. } \\
83\end{array}$ \\
\hline Thraso Kastanakis & $\begin{array}{l}\text { Dansoz Kontesina Feliçita } \\
\text { (La danseuse Kontesina Felicita) }\end{array}$ & $\mathrm{N}$ & 18 & $\begin{array}{l}1^{\text {er }} \text { avr. } \\
34\end{array}$ \\
\hline $\begin{array}{l}\text { Grigorios } \\
\text { Xenopulos }\end{array}$ & $\begin{array}{l}\text { Olüm Kar Isında } \\
\text { (Face à la mort) }\end{array}$ & P. TH & 19 & $\begin{array}{l}15 \text { avr. } \\
34\end{array}$ \\
\hline Tatiana Stavrou & $\begin{array}{l}\text { Ne Hale Gelmişti ? } \\
\text { (Dans quel état se trouvait-il ?) }\end{array}$ & $\mathrm{N}$ & 24 & $\begin{array}{l}1^{\text {er }} \text { juil. } \\
34\end{array}$ \\
\hline
\end{tabular}




\begin{tabular}{|c|c|c|c|c|}
\hline Alexandros Baras & $\begin{array}{l}\text { Yüzük } \\
\text { (L'anneau). }\end{array}$ & $\mathrm{N}$ & 37 & $\begin{array}{ll}1^{\mathrm{er}} & \text { oct. } \\
34 & \end{array}$ \\
\hline Katina Papa & $\begin{array}{l}\text { Dünya' } i^{9} \\
\text { (Le monde) }\end{array}$ & $\mathrm{N}$ & 43 & $\begin{array}{ll}15 & \text { avr. } \\
35 & \end{array}$ \\
\hline Kostis Bastyas ${ }^{10}$ & $\begin{array}{l}\text { Deniz } \\
\text { (Lamer) }\end{array}$ & $E$ & 45 & $\begin{array}{ll}15 & \text { mai } \\
35 & \end{array}$ \\
\hline $\begin{array}{l}\text { Yorgos } \\
\text { Varopoulos }\end{array}$ & $\begin{array}{l}\text { Keman } \\
\text { (Le violon) }\end{array}$ & $\mathrm{N}$ & 50 & $\begin{array}{l}1^{\mathrm{er}} \text { sept. } \\
35\end{array}$ \\
\hline Petros Haris ${ }^{11}$ & $\begin{array}{l}\text { Beklenilmeyen Kitap } \\
\text { (Le livre que l'on n'attendait pas) }\end{array}$ & $\mathrm{N}$ & 65 & $\begin{array}{l}15 \text { mars } \\
36\end{array}$ \\
\hline $\begin{array}{l}\text { Konstandinos } \\
\text { Cavafis }\end{array}$ & $\begin{array}{l}\text { Mumlar, Kaleler ve Dokuzdan Beri (Les bougies, les } \\
\text { forteresses et depuis neuf heures) }\end{array}$ & $\mathrm{P}$ & 70 & $\begin{array}{ll}15 & \text { mai } \\
36 & \end{array}$ \\
\hline $\begin{array}{l}\text { Dimostenis } \\
\text { Voutiros }\end{array}$ & $\begin{array}{l}\text { Hirslz } \\
\text { (Le voleur) }\end{array}$ & $\mathrm{N}$ & 74 & $\begin{array}{l}1^{\mathrm{er}} \text { août } \\
36\end{array}$ \\
\hline Glatkos Alitersis & $\begin{array}{l}\text { Cellat } \\
\text { (Le bourreau) }\end{array}$ & $\mathrm{N}$ & 88 & $\begin{array}{l}1^{\mathrm{er}} \text { mars } \\
37\end{array}$ \\
\hline Yannis Stefanakis & $\begin{array}{l}\text { Güllü Ev } \\
\text { (La maison aux roses) }\end{array}$ & $\mathrm{N}$ & 95 & $\begin{array}{ll}15 & \text { juin } \\
37 & \end{array}$ \\
\hline $\begin{array}{l}\text { Eli Aleksiou } \\
\text { Daskalakis }\end{array}$ & $\begin{array}{l}\text { Üç Kardeş } \\
\text { (Trois frères) }\end{array}$ & $\mathrm{N}$ & 130 & $\begin{array}{l}1^{\mathrm{er}} \text { déc. } \\
38\end{array}$ \\
\hline
\end{tabular}

$R=$ roman, $N$ = nouvelle, $\mathrm{P}$. $\mathrm{TH}$ = pièce de théâtre, $\mathrm{P}=$ poésie

6 Pendant la Seconde Guerre mondiale, aucun texte traduit du grec ne parait dans la revue. Faut-il attribuer cette absence à la crise internationale ou tout simplement à la disparition d'Avram A. Papazoğlu? En attendant que d'autres recherches fournissent une réponse satisfaisante à cette question et tracent la biographie d'Avram N. Papazoğlu, on peut dire que ce personnage quelque peu énigmatique a joué entre les deux guerres un rôle très intéressant pour rapprocher les deux cultures, grecque et turque et surtout pour faire connaître les littératures réciproques sur les deux rives de la mer Égée. À l'étude du tableau, on le constate, c'est lui qui a traduit la totalité des œuvres littéraires grecques parues dans Varlk. Mais il a surtout traduit des œuvres des écrivains turcs en grec dans la revue Néa Estia qui d'après Papazoğlu ${ }^{12}$ a publié régulièrement et tous les quinze jours des articles sur les mouvements intellectuels et littéraires turcs, tout en offrant des extraits d'œuvres littéraires. Il a aussi traduit l'ouvrage intitulé Damla Damla (Goutte à goutte) ${ }^{13}$ de Ruşen Eşref (Ünaydım) (1892-1959) ${ }^{14}$ alors ambassadeur de Turquie à Athènes (1934-1939). Son anthologie de la littérature turque fut éditée par la maison d'édition Andonopoulos. De même, le lecteur grec connaitra l'un des chroniqueurs ottomans, Tursun Bey, ainsi que le voyageur ottoman le plus célèbre Evliya Çelebi, grâce aux traductions de Papazoğlu. Sa philosophie fondamentale était de rapprocher les deux peuples par la littérature et la culture et surtout de désigner et dénoncer les responsables des différends entre Grecs 
et Turcs. Dans cette période de réformes, d'anticléricalisme et du positivisme, Papazoğlu clame que "sans l'influence néfaste des popes et des hocas (religieux musulmans), qui a causé la haine et le mécontentement entre les deux peuples, les Grecs et les Turcs auraient continué à vivre ensemble en Anatolie $»^{15}$. Toujours dans cette période d'entre-deux-guerres, les revues Çığır, Gündüz, Ar et Dârülbedâyiit ont donné aussi des traductions de la littérature grecque, comme des informations et analyses sur les mouvements littéraires en Grèce. Dans cet élan de traduction de la littérature grecque contemporaine, la contribution de l'idéologie kémaliste sur l'appropriation de la terre anatolienne ou du « nationalisme territorial » est indéniable. Mais le mouvement littéraire "néo-hellénisme » (Nev Yunanilik) inauguré en 1911 par Tevfik Fikret (son poème Prométhée en est la meilleure illustration) et développé par Yahya Kemal et Yakup Kadri en fut encore plus déterminant. Les œuvres des représentants les plus emblématiques de ce courant poétique ont connu quelque succès dans cette période d'entre-deux-guerres: Salih Zeki Aktay (1896-1970), Ali Mümtaz Arolat (1897-1967) et Mustafa Seyit Sutüven (1908-1969). Plus tard, dans les années quarante, Süküfe Nihal Başar, Ahmed Hamdi Tanpınar, Can Yücel ou encore Melih Cevdet Anday continuent dans cette tradition néo-hellénique (ou dite encore «mythologique ») qui contribue à établir des ponts entre l'Antiquité anatolienne et la Turquie contemporaine.

7 Cela dit, même si la revue Varlı a continué à publier certaines nouvelles et quelques poèmes, c'est au ministère de l'Éducation nationale (1938-1946) de Hasan Ali Yücel (1897-1961) que la Turquie doit la véritable révolution de la traduction. Fervent défenseur de la philosophie et de la littérature classiques grecques, Yücel a fait traduire pratiquement tous les textes principaux de la période hellénistique. Convaincu que la civilisation contemporaine occidentale a pour source la Grèce ancienne, Yücel, enfant des lumières européennes, a fait publier la revue Tercüme, mais surtout plusieurs centaines d'ouvrages (environ 900) de la période classique occidentale, puis orientale pour le compte de son ministère, tout en subventionnant dans le secteur privé les traductions des œuvres contemporaines. Dans le premier numéro double et spécial de la revue Tercüme consacré aux textes classiques grecs (Homère, Hésiode, Eschyle, Euripide, Aristophane, Hérodote, Thucydide, Platon, Xénophon, Aristote, Lysias, Isocrate, Lycurgue), Hasan Ali Yücel affiche clairement l'ambition républicaine de faire sienne la Grèce ancienne :

"Il faut énoncer comme une donnée absolue et comme une foi commune de l'humanité progressiste que la source principale de la civilisation que nous appelons occidentale, celle que nous sommes obligés d'adopter, réside dans la Grèce ancienne »"

De même :

"Les ceuvres anciennes des Grecs dont nous avons hérité sur le territoire anatolien, comme celles de tous ceux qui sont passés par là sont les nôtres. Les anciens Grecs n'étaient peutêtre pas nos citoyens, mais ils étaient nos compatriotes $»^{18}$.

8 On ne peut pas trouver déclaration de foi plus claire pour revendiquer l'héritage grec de l'Asie Mineure comme fondement de la civilisation turque contemporaine.

\section{La littérature grecque en Turquie : poésie et prose}

9 Passée la période romantique et volontariste, les milieux littéraires et éditoriaux turcs se sont mis à traduire à un rythme régulièrement en augmentation des œuvres grecques. Selon une étude inédite réalisée pour la période entre 1961 et $2002^{19}$, les 
œuvres grecques en prose traduites en turc intéressent essentiellement le passé et le présent de la Turquie et ces traductions sont faites par ceux qui n'approuvent pas la pensée officielle du régime en place ; autrement dit, généralement ce sont des auteurs et traducteurs de gauche et des maisons d'éditions de cette tendance qui s'attellent à diffuser cette littérature. Durant la période considérée, cent dix-neuf ouvrages de cinquante-deux auteurs grecs furent publiés. La plupart de ces auteurs disparaissent après une ou deux éditions. Inversement, Ritsos, Kazantzakis ou encore Cavafis, Samarakis ont eu respectivement de treize à six éditions.

\section{La poésie d'abord}

10 Mais le genre littéraire le plus traduit du grec en turc est la poésie. En premier lieu, l'écho de la poésie moderne grecque fut grand en Turquie et surtout après l'attribution du prix Nobel en 1963 à Giorgios Seféris (1900-1971). Ce poète originaire d'Izmir et chantant l'exil, sa maison et sa terre natales, retrouvera une Turquie nouvelle et visitera sa ville, alors qu'il est ambassadeur de Grèce à Ankara (1948-1950). Mais surtout, dans le contexte de la nouvelle constitution démocratique des années 1960, la traduction de la littérature grecque a pris son envol avec les auteurs de cette fameuse période que l'on appelle "Décadentisme " et notamment Constantin Cavafis, Nikos Kazantzakis. Autant que l'on puisse savoir, le premier poème de G. Seféris est traduit en turc en 1961 par Nermin Menemencioglu dans la revue Yeditepe ( $\left.{ }^{\circ} 45\right)$. Par la suite, l'auteur fut traduit partiellement de l'anglais par Cevat Çapan ${ }^{20}$, puis du grec par Herkül Millas $^{21}$. De la même manière, Odysséas Élytis, prix Nobel, lui aussi, en 1979, est naturellement très connu en Turquie et d'abord grâce à son recueil L'arbre de lumière (1971) traduit en turc sous le titre L'Arbre fou de Grenadine (Çılgin Nar Agacı).

11 Dans les années 60 et 70, non seulement la revue Varlık a joué son rôle habituel de promotion des littératures étrangères en Turquie, mais d'autres revues comme Türk Dili ${ }^{22}$, Yeditepi ${ }^{23}$, Yeni Ufuklar ${ }^{24}$, Cep $p^{25}$, Yeni, Milliyet Sanat, Yazko Çeviri (premier numéro: août $1981^{26}$ ) ont pris le relais et ont publié certains poèmes grecs des auteurs aussi connus que Cavafis, mais aussi moins connus, tels que Petros Chronas ou encore Sikelianos. Désormais, les maisons d'édition participent assez activement à la publication des poèmes des auteurs grecs. Ainsi, les premiers recueils de poèmes de Cavafis sont édités sous les titres «En attendant les Barbares » (Barbarları Beklerken), par Imge Yayımları, en 1981, puis sous forme d'anthologies : Kavafis'ten Kırk şiir (1982). En 1981, Yannis Ritsos a été à l'honneur : traduit par Cevat Çapan (10 poèmes) et Özdemir İnce (13 poèmes), il fut publié dans Yazko Çeviri $\left(\mathrm{n}^{\circ} 1\right)$. Dans cette période, la poésie grecque venait en troisième rang dans les traductions de poésie après la française et l'anglo-américaine; en 1982, en particulier, dans une statistique concernant la traduction de la poésie étrangère en turc, la poésie grecque venait en troisième position après la poésie française ( 59 poèmes), polonaise ( 40 poèmes à cause de l'attribution du prix Nobel en 1981 à Czeslaw Milosz) puis grecque (32), mais avant la poésie allemande (28), italienne (22), hongroise (17), américaine (16), etc. et seulement trois d'un pays arabe (Maroc: 3 ) sans que nous sachions si ce sont des poèmes en arabe ou en français ${ }^{27}$.

12 Dans une autre enquête faite en 1984, on a demandé à vingt et un principaux auteurs turcs ce qu'ils avaient retenu de l'année passée parmi les livres publiés, poèmes ou romans, œuvres originales de la littérature turque comme traductions. Quatre d'entre 
eux ont choisi des œuvres de la littérature grecque : Erhan Bener a aimé les poèmes réunis sous le titre de Ardıç Kuşu de Séféris traduit par Herkül Millas ; Muzaffer Burukçu a considéré comme son livre de chevet, le recueil de d'Élytis Odysséas, Çılgin Nar Agacı. Bener pense que ce livre est "la synthèse des oppositions entre la réalité et l'extraordinaire. Élytis voit la nature avec un regard différent, mais admiratif, lui permettant de créer une œuvre de haute qualité. Selon lui, Çılgın Nar Agacı est un festival de cris, de sens, de couleurs, il est rempli d'un effort noble transformant comme un magicien la poésie en réalité qui, elle, devient poésie dans la tourmente de changement d'identitée ${ }^{28}$. Aziz Çalışlar a consacré une partie de son année à la poésie grecque. Non seulement il a lu et apprécié Çağdaş Yunan şiiri Antolojisi (Anthologie de la poésie grecque contemporaine), faite par Cevat Çapan, mais aussi Çılgın Nar Agacı et de quarante poèmes de Cavafis. Les traductions de Cevat Çapan sont pour lui un vrai plaisir, et la caractéristique principale de la poésie grecque est l'accord parfait entre l'ego du poète grec, l'univers (la nature) et l'histoire, sa fusion avec ces deux éléments pour trouver l'écho: «on peut dire un écho philosophique " ${ }^{29}$. De la même manière, Necati Güngor a lu plusieurs fois Élytis dans la traduction de Cevat Çapan dont le travail « ne sent pas la traduction». Bien entendu, pour avoir une courbe à long terme, ce type d'enquête doit être poursuivi et élargi afin de voir l'audience des auteurs grecs contemporains auprès des intellectuels turcs, mais a priori ils ont un capital de sympathie auprès de ceux-ci que d'autres auteurs de la région sont loin d'atteindre.

\section{Yannis Ritsos : le mythe}

13 Ayant grandi dans l'atmosphère déprimante des années 20-30, Yannis Ritsos (1909-1990) est certainement le poète grec le plus admiré en Turquie. On peut expliquer cet engouement pour lui, le représentant le plus éminent de la poésie grecque contemporaine, à cause de ses vers brefs, ses phrases entrecoupées, rappelant un Yunus Emre, mais aussi parce qu'il est le symbole, au même titre que Nâzım Hikmet, de la résistance passive contre l'oppression des dictatures de Métaxas et de Papadopoulos ; il est la conscience nationale, le défenseur de la classe ouvrière. Sous la plume de Cevat Çapan, son poème de 1937, la Chanson de ma sour (Kızkardeşimin Tüküsü) devient le drapeau de intellectuels dans les années 70 contre le régime turc. En effet, Ritsos, le " poète engagé » a une place particulière auprès des élites littéraires de gauche en Turquie : ses écrits sont sanctionnés par le régime du dictateur Métaxas, son recueil Épitaphe est brûlé sur la place publique; durant la Seconde Guerre mondiale, il est persécuté en tant que membre du parti communiste et de ce fait il connaîtra les camps de "rééducation ». Autant de moments forts qui le désignent aux yeux des intellectuels turcs persécutés comme le symbole de la liberté d'expression et le modèle à suivre. « Le plus grand poète contemporain ", selon Aragon, Yannis Ritsos sera connu en Turquie et par les auteurs turcs à travers les Lettres Françaises. Son hellénisme ou sa "grécité » ne dérange nullement, au contraire, ses écrits sur la mythologie grecque, ses cris à la fois de révolte et d'appel à la tolérance, son questionnement sur la guerre, le sens du pouvoir, ses écrits où les temps se mêlent et où la chronologie « objective " se distord pour ne laisser place qu'au cursus de la pensée poétique correspondent bien aux préoccupations des intellectuels turcs de «ces années de plomb» en Turquie où les particularismes politiques et ethniques sont condamnés violemment par les pouvoirs civils, mais aussi militaires. 
14 Un autre élément de taille lie Ritsos à la poésie turque « engagée », lui et Nâzım Hikmet mènent le même combat à travers leur poésie. Non seulement Ritsos a rencontré plusieurs fois Nâzım Hikmet, mais, surtout, il avait une admiration sans bornes pour lui et réciproquement. C'est la raison pour laquelle Nâzım Hikmet fut traduit en grec par Ritsos et son ouvrage plusieurs fois réédité ; probablement le plus beau poème écrit pour Nâzım Hikmet appartient également à Ritsos :

Lorsqu'un prénom est devenu musique et univers

À Nâzım Hikmet mon frère Nâzım Nâzım aux yeux bleus

avec ton cœur bleu

Tes songes encore plus bleus

toi, lorsque tu regardes l'obscurité profondément

sans éprouver la moindre haine

tu transformes en bleu l'obscurité

Nâzım

toi, avec un verre de vin

avec le genou d'une belle femme

avec un coin de mer sur lequel est arboré le drapeau du peuple

tu illumines les horizons

là où tout est détruit

des rochers s'ébranlent avec liesse des collines jusqu'aux barques

et sous les lampadaires de rue

un chien se perd dans ses pensées

Nâzım

j'ai vu tes petits musiciens de rue

sur le pont de Galata

quelques vers à toi étaient cachés

dans les boites des violons

hormis ceux qu'ils avaient l'autorisation de chanter

ils attendaient en regardant les nuages

le jour où ils pouvaient les chanter

(Nâzım, parfois, un violon est comme un poing serré

et dans le poing serré est caché un canal)

Nâzım

j'ai vu les ouvriers grévistes des docks

parmi les grues, les piliers, les poèmes

les sacs, les caisses et les roses

à côté du grand navire

deux lumières qui attendaient

le navire sur le point de lever l'ancre

(qui sait vers quelle direction?)

c'était un combat, c'était une passion,

toi, Nâzım, tu étais son capitaine

Se dirigeant au-delà des frontières

ce voyage

Nâzım

quelqu'un montait par la passerelle du navire avec ses canaris

[dans la cage

ses chaussures aux lacets défaits

au lieu de dire "bonjour "

"rouge", dit ce quelqu'un

une femme pleurait devant la porte

le pêcheur est passé

sans que personne ne le remarque

dans sa montre

sous le verre poussiéreux 


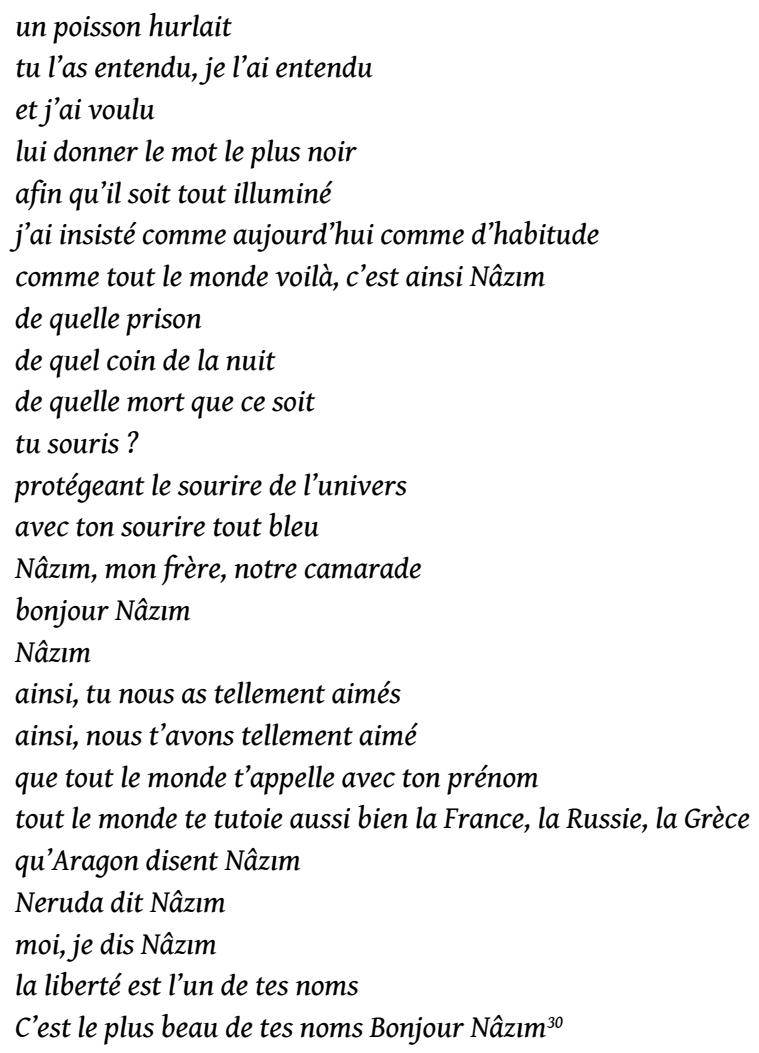

15 L'irruption de Ritsos dans la vie littéraire turque ne fut possible que grâce au travail du poète Özdemir İnce (1936-) qui, avec ses traductions à partir du français, a eu le prix de Türk Dil Kurumu (la Société de langue turque) en 1979 avec les Pierres, répétitions, barreaux (Taşlar, Yinelemeler ve Parmakliklar). Quant à Cevat Çapan, auteur d'une anthologie de poésie grecque, il est aussi l'un des traducteurs de Ritsos, cette fois-ci à partir de l'anglais. Selon les dires de İnce, Ritsos fut connu d'abord par quelques initiés seulement dans les années 1965-1966, mais à partir de 1971 lorsque ses poèmes seront publiés en turc, il ne quittera plus les vitrines. Dans la décennie 70, İnce se consacre en grande partie à traduire son œuvre. Cette obsession va même plus loin, car il craint de perdre son propre style au bénéfice de celui de Ritsos. et lui exprime cette crainte, au cours d'une de leurs nombreuses rencontres ${ }^{31}$. Si l'on en croit l'un de ces entretiens, le poète pensait aussi que le français était une langue ossifiée et qui ne pouvait pas rendre compte de sa pensée, tandis que le turc était une langue jeune correspondant mieux aux exigences de l'auteur.

Il faut ajouter enfin que jusqu'à une date récente le seul site internet au monde consacré à Yannis Ritsos était fait par les Turcs et en turc, où biographie, poèmes, photos côtoyaient les entretiens qu'auteurs et journalistes avaient réalisés avec le poète.

\section{Deux chantres de la Crète : Kazantzakis et Prévélakis}

17 Assurément, le romancier grec qui est le plus traduit et lu en Turquie est Nikos Kazantzakis (ou Kazancakis en turc). Contrairement à la chronologie avec laquelle Kazantzakis a écrit ses romans, les traductions en turc ont paru par le dernier (Lettre au Greco, posthume, 1961). Sur la couverture, l'éditeur E. Yayımları n'a pas hésité à noter : «Un maître, une vie croquée à pleines dents, une narration qui règle ses comptes avec 
son temps. Ses souvenirs et pensées pétries dans la chaleur humaine, malaxées avec l'air de la Crète, baignées dans la mer Égée ». La publication de cet ouvrage qui est une autobiographie imaginaire, ou, même idéalisée est présentée par l'éditeur turc comme une confrontation entre lui-même et tous les domaines de l'existence : la colère, la religion, la nature, la création, Jésus, la philosophie sont présentés sous des formes très personnelles, c'est-à-dire avec sa propre expression, «d'un œil crétois». C'est cet aspect crétois qui est mis également en exergue dans le Capitaine Mihalis, cette fois-ci publié par Can Yayımları, comme le reste des ouvrages de Kazantzakis traduits en turc, et qui est présenté au lecteur comme étant «le chef d'œuvre d'un des plus grands maitres de notre temps, chef d'œuvre qui sera compris le mieux par les lecteurs turcs ».

Ainsi, comme on le voit, Kazantzakis est connu en Turquie plutôt comme un romancier que comme un poète. Ses principaux romans Zorba (Alexis Zorba) ${ }^{32}$, Günaha Son Çagrı (La dernière tentation, 1954), İsa Yeniden Çarmiha Geriliyor (Le Christ Recrucifié, 1954), Allahın Fikarası (Le pauvre de Dieu, 1957), Ya Hürriyet Ya Olüm (La liberté ou la mort, 1967), İspanya Yaşasın Olüm (Voyages III, Espagne, 1968), Toda Roba (Toda Raba, 1969), Kardeş Kavgası (Les Frères ennemis 1989), Kayalı Bahçe (Le jardin des Rochers, 1990), Assisli Francis (Le pauvre d'Assise, 1995) sont traduits en turc et ont fait l'objet de nombreuses éditions. Comme chez Pandélis Prévelakis, la Crète est chez Kazantzakis probablement le personnage le plus important et les éditeurs turcs en tirent parti, car dans l'imaginaire et l'histoire turques, la Crète a une existence réelle et particulière : les très nombreuses histoires racontées à propos de l'île, sans parler des populations issues du grand « marchandage humain " du traité de Lausanne ne peuvent que rendre attrayants les romans de Kazantzakis. Le directeur des Éditions Can Yayımları ne s'y trompe pas et présente le Capitaine Mihalis en ces termes :

"Kaptan Mihalis, en tant que personnage de roman, est un type réel local, vivant, en chaire et en os, et les pieds solidement posés sur terre, c'est un véritable résistant populaire. Nous croyons que ce sont les lecteurs de notre pays qui comprendront le mieux Le Capitaine Mihalis qui est l'œeuvre principale de l'un des plus grands maîtres de la littérature de notre temps".

19 Ainsi, le capitaine Mihalis est comparable à ces figures d'hommes anatoliens mythiques et romanesques dont le courage, le franc-parler, la force physique et de caractère ont fait l'objet de la littérature turque épique.

De même, si Pandelis Prévélakis avec son Girit'te Bir Sehrin Hikayesi/Histoire d'une ville en Crète (To Kroniko Mias Politeias ${ }^{33}$ ) a trouvé un écho en Turquie, c'est parce que son ouvrage parle d'une ville crétoise mieux que quiconque, répondant à la nostalgie du lecteur turc potentiel qui trouverait son compte dans ces phrases d'A. Chamson:

"Réthymnon est comme une personne vivante. Elle a sa mémoire et ses rêves, ses désirs et ses regrets. Elle peut souffrir; elle peut aussi être heureuse. Elle existe cependant, c'est à travers le conteur, à travers Prévelakis que nous prenons acte avec elle. Il nous parle de Réthymnon comme d'une femme aimée, aimée si profondément et depuis si longtemps qu'il peut la comprendre et la juger sans manquer à cet amour. C'est un amour si total et sûr de lui qu'il a dénoué son bandeau. Ce livre est à deux personnages: la ville et l'auteur qui forment comme un couple et semblent se tenir par la main ${ }^{34}$.

Maisons de style ottoman à Réthymnon 


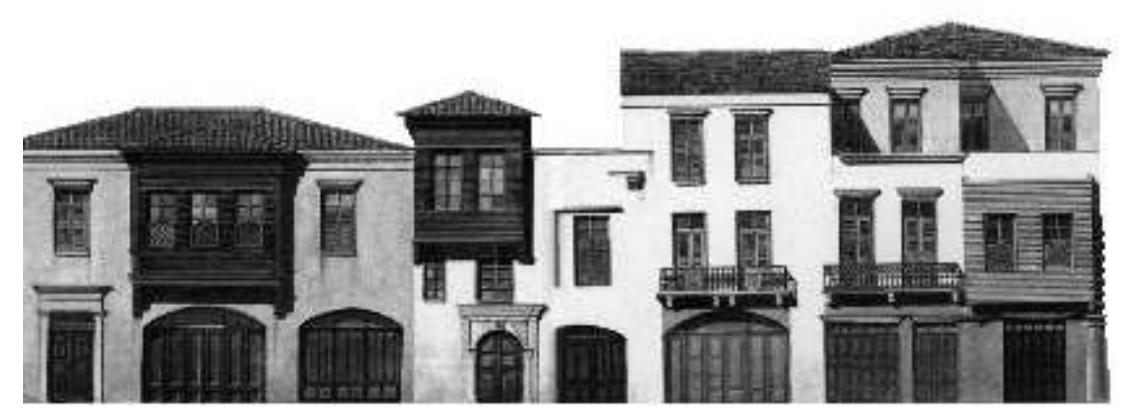

http://users.swing.be/voygr/voygr/0404.htm (consulté le 04/07/2012)

21 Les influences turques sont encore visibles sur les façades de nombreuses maisons urbaines. L'élément le plus typique est le xostego, balcon clos en bois, typiquement balkanique, dont la construction fut encouragée par les autorités ottomanes.

Prévélakis montre avec brio les grandes déceptions de la population musulmane de Crète, forcée par les politiques à quitter sa terre natale, après la Première Guerre mondiale, au moment où elle pensait que tout allait rentrer dans l'ordre: les gens avaient retapé leurs maisons, cultivé leur terre et un jour ils ont reçu l'ordre de tout abandonner. Le livre de Prévélakis est un hymne à la cohabitation des populations chrétiennes et musulmanes de Crète.

\section{À la recherche du temps perdu : la collection Marenostrum et les autres}

\section{La collection Marenostrum}

23 La collection Marenostrum est née également de cette philosophie de la cohabitation sur les deux rives de la mer Égée et maudit les auteurs du nettoyage ethnique et religieux du début du XXe siècle. Initiée par Aye Nur et Ragip Zarakolu aux Éditions Belge (créées en 1977), cette collection a contribué à la diffusion de la littérature grecque en Turquie et a valu à leurs créateurs le prix Abdi İpekçi ${ }^{35}$. Dans cette collection, la Méditerranée, contrairement à son sens romain, est considérée comme la mer commune de toutes les cultures et de tous les peuples qui l'entourent et non pas le bien d'une seule nation. En somme, les éditeurs lui donnent un sens braudélien :

"Cette mer que nous partageons, où nous nous battons, où nous aimons, où nous mourrons, celle qui nous lie étroitement, celle qui est pleine de souvenirs communs, celle qui est chargée de tous les sentiments, de tous les enthousiasmes et des excès; nous pensons que les mers Adriatique, Égée, Marmara et la mer Noire sont ses bras. La collection Marenostrum est un concert de peuples, et notre voyage commence avec Iordanidou, par Istanbul et continue avec d'autres auteurs sur d'autres ports, îles, chaînes de montagnes courant derrière la mer, en passant par les Balkans, par le Caucase... cette série sera spéciale : avec nos lecteurs, nous chercherons les traces de l'atmosphère fraternelle du temps perdu que l'on a voulu enterrer dans les ténèbres de l'histoire; nous établirons un pont spirituel pour un avenir commun, pour sauver notre mer $»^{36}$.

Ainsi, cette collection a pour but de réunir et de présenter parfois pour la première fois les auteurs prosateurs grecs qui sont arrivés juste après la Première Guerre mondiale. Ces ouvrages de qualité et de succès en Grèce en leur temps constituent la production de toute une génération d'écrivains antimilitaristes dans une atmosphère déprimante, 
« débilitante » diraient certains. Cette littérature est marquée par la période de 1922, par cette "Catastrophe de l'Asie mineure" suivie par les déplacements des populations. Elle est généralement frappée par un climat qui porte tout le poids du désastre militaire grec en Anatolie face aux Turcs. Un des premiers prosateurs de l'après-guerre Angélos Terzakis évoque cette période dans ces termes :

"Je revois la vie à travers les vitres opaques d'un café athénien, devenues roses au crépuscule; une vie athénienne désormais disparue. Dehors, dans la rue, où il y avait tant de poussière et si peu de mouvement, les rares passants marchent sans hâte. Quelque part, dans le coin, chante un orgue de Barbarie, avec de gros sanglots. Une odeur de nourriture qu'on fait cuire monte dans le quartier. Parmi les ombres floues de la nuit qui tombe, on voit soudain passer dans la rue une bande de gens silencieux: ce sont les réfugiés de la Catastrophe".

"On a dit que c'était l'époque des vaincus. Ceux qui l'ont dit étaient ceux qui sont venus un peu plus tard, reposés, au costume bien repassé, coupé chez quelque tailleur d'Europe occidentale. Des vaincus? Je préférerais dire "du drame ». Il y a des époques d'insouciance et des périodes d'épouvante; des temps où la conscience est endormie et d'autres où l'âme est en état d'alerte. Ces années qui s'écoulèrent entre 1922 et 1929 furent faites d'un étrange mélange d'insouciance et de préoccupations, de présages et de songes. L'Athènes la plus vivante avait pour note dominante d'être petite bourgeoise: c'était une vaste zone qui côtoyait la rue Stadiou ; c'était encore un calque fidèle de la province ».

Cette génération des années 30 comprend des écrivains en majorité originaires de l'exEmpire ottoman ou ceux qui y ont vécu. Ils décrivent avec beaucoup de détails et de nostalgie les villes et les provinces où ils ont habité (Istanbul, Izmir, Ayvalık, etc.) et les événements historiques. Ces auteurs ont créé une mémoire historique en Grèce, relative au paradis perdu qui serait l'autre côté de la mer Égée.

Les caractéristiques communes de ces ouvrages consistent à la recherche d'une conciliation entre les deux rives de la mer Égée : si les deux peuples, turc et grec, sont arrivés là où ils sont, ce n'est que la faute des étrangers, des impérialistes. Le sentiment général est que les Turcs ont été trompés par les Allemands et les Levantins au début de ce $\mathrm{XX}^{\mathrm{e}}$ siècle, et que les perdants en sont les populations simples, les paysans et les ouvriers.

Nous sommes à la veille de la guerre balkanique de 1912 ; Dido Sotiriou dans ses Terres ensanglantées conforte ce postulat:

«Tu penses, Manoli, que les patrons ont quelque chose à craindre? Ce soir pour un bout de papier, des navires immenses, des wagons entiers remplis de coton, de cuirs, de tabac seront vendus. Occupe-toi de toi. Eux, ils ont des montagnes, des villages, des mines, des usines... Ils ne se soucient pas du tout de l'État! Ils achètent les fonctionnaires pour qu'ils ferment les yeux sur leurs saletés... Le pacha caresse ses billets de banque de cinq, la police ses pièces de

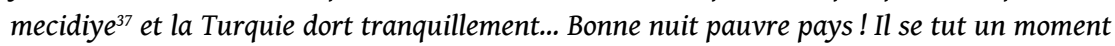
puis rajouta:

- Nos vrais ennemis, ce sont les Levantins, et les sangsues qui sucent le sang de la Turquie de sa source... Je ne sais pas d'où ils sont venus pour nous planter les dents dans notre peau... Ce sont des poux -- que Dieu les maudisse --, et tu verras, notre catastrophe viendra de ces genslà, mais pas des Turcs $»^{38}$.

L'auteur le plus emblématique de cette génération des années 30 est Maria Iordanidou. Née en 1897 à Istanbul, puis partie en Grèce, elle revient à Istanbul pour poursuivre ses études au Robert collège, après avoir passé une bonne partie de son enfance au Pirée, près d'Athènes. En 1914, elle va à Batoum et après cinq ans de séjour en Russie, elle rentre à Istanbul, pour en repartir vers Alexandrie. En 1923, elle s'installe à Athènes, son premier roman Loxandra, retour d'Istanbul est édité en 1963, alors qu'elle avait 63 
ans. Ce premier livre sera traduit et édité à Istanbul seulement en 1989 au moment de sa mort à 92 ans. Puis, Çılgin Kuşlar Gibi (Comme des oiseaux fous), Felegin Çemberinde (Dans le cercle du destin), Bizim Avlu (Notre cour) le suivront.

Dido Sotiriou est un autre écrivain, aussi originaire d'Anatolie (Aydın) dont les ouvrages sont traduits en turc. Notamment, I nekri Perimenun (Les morts attendent), avec quarante ans de retard, en 1995. Mais Matomena Homata ou Benden selam Soyle Andolu ya (traduit du français par Attila Tokatlı) est son œuvre de témoignage d'un personnage réel, Manoli Axiotis, qui a été réquisitionné pendant la Première Guerre mondiale pour le travail obligatoire dans les travaux publics, s'est battu contre les Turcs aux côtés de l'armée grecque, est fait prisonnier, puis envoyé en camp. Il s'agit dans ce roman de peindre un monde disparu, la cohabitation anatolienne des Grecs et des Turcs. Sa thèse principale est que, ces populations que l'impérialisme et le capitalisme occidentaux ont perturbées vivaient en paix.

Dans ces ouvrages, la similitude des thèmes et du style avec ceux de Sait Faik est certainement un élément qui attire par ailleurs le lecteur: le cosmopolitisme, les relations quotidiennes des diverses communautés anatoliennes dans le climat douillet des villes côtières. Ainsi dans la couverture de Ege Hikayeleri (Histoires égéennes) ${ }^{39}$ de Ilias Vénézis on peut lire: «On peut parler d'une similitude entre les œuvres de Sait Faik et celles de Vénézis. Même si tous les sujets ne coïncident pas, leur style, la manière de se pencher sur les subtilités des relations humaines et leur façon de mettre en mots les finesses difficilement descriptibles de la psychologie humaine font penser à cette similitude. Comme ceux de ces livres, les personnages des livres de Vénézis sont des gens simples, comme les pêcheurs, les hommes ordinaires et les immigrés venus d'Anatolie. Dans ces nouvelles, on lit souvent la tristesse et le désespoir. Vénézis, en s'adressant aux Turcs dit: "Nous avons un mot à dire à ceux qui vivent sur la rive orientale de la mer Égée: Si vous voulez que nous oubliions nos épopées et nos martyres, cela, on ne peut le faire. Mais nous pouvons faire autre chose, autrement plus glorieuse et plus importante: nous pouvons ne pas être rancuniers. Sans faire abstraction nous pouvons réaliser la fraternité de nos deux peuples; nous pouvons mettre d'un côté de la balance tout ce que nous avons enduré, tout le poids de tant d'années, notre arrachement à notre patrie, et de l'autre, notre amour pour la paix, la conscience qu'il ne faut plus jamais que nos peuples fassent la guerre, ne tentent plus de supprimer l'autre... ».

31 Ainsi, une trentaine d'ouvrages des auteurs grecs des années trente ont paru dans cette collection : Stratis Tsirkas (Bomba Nurettin, Nil Vadisinden); Menis Kumandareas ${ }^{40}$ (Eski Tüfekler), Thrasos Kastanakis ${ }^{41}$ (Hacı Manuil, 1995) ; Stratis Myrivilis (Arnavut Vasili, 1997) et bien d'autres.

\section{La mer Noire ou le sous-développement littéraire?}

Si les deux rives de la mer Égée ont fertilisé les inspirations littéraires, la mer Noire qui a fourni une bonne partie de la population grecque actuelle et alimente encore les passions locales est loin de constituer un thème littéraire privilégié en Grèce. La collection Marenostrum s'est donc engouffrée dans la petite brèche qu'elle a trouvée en la personne de Yorgos Andreadis, un auteur de province, totalement ignoré par les anthologies littéraires. Primé par la fondation Abdi İpekçi pour son livre Tamama, Andreadis, une sorte de conteur-reporter, se consacre à la mer Noire et à la culture 
pontique et figure à ce titre dans le répertoire de Marenostrum avec une série de cinq petits ouvrages.

La thèse principale de cet auteur, qui représente d'ailleurs un certain courant idéologique minoritaire en Grèce du Nord, est que les habitants actuels de la mer Noire seraient en grande partie des crypto chrétiens, convertis donc d'apparence à l'Islam par pure tactique, mais sans véritablement croire à cette religion. En somme, ils seraient des sortes de Maures ou de Marranes chrétiens. Y. Andreadis visite (ou visitait) très souvent la Turquie, et glane quelques histoires de la période de la fin du début du $\mathrm{XX}^{\mathrm{e}}$ siècle, qui concernent les victimes de la période d'échange de population, des familles divisées. L'histoire de Tamama est précisément celle d'une fillette séparée de sa famille en 1918 et finalement retrouvée en 1973. Elle est attestée comme une histoire vraie et qui a ses ramifications en Turquie comme en Grèce. Le livre se termine dans ces termes :

«N'oublions pas non plus les nombreux anonymes qui ne sont pas restés indifférents devant les souffrances de leurs frères hommes dans les tourmentes de la guerre. La fraternité entre les peuples ne peut être fondée que sur la grandeur et la sagesse de ce genre de personnes, cette fraternité universelle qui est l'assurance de l'amitié entre les peuples $»^{42}$.

Visiblement, avec ses recherches, Y. Andreadis irrite les autorités turques et, depuis 1999, il est déclaré en Turquie persona non grata et considéré comme« un activiste ayant pour but de créer un État pontique sur la mer Noire », il est toujours interdit de voyage et de séjour en Turquie. Ce qui montre encore une fois les difficultés de publier des œuvres grecques en Turquie.

\section{Censure ou autocensure?}

35 C'est d'ailleurs ici qu'il faut parler du problème de la censure ou de l'autocensure dans les traductions. Traduire la littérature grecque en Turquie n'a jamais été un acte facile ni innocent. Lorsqu'un auteur œuvre pour la réconciliation des deux peuples, la traduction de sa production ne pose pas de problème particulier en Turquie. Mais dès que l'on touche à des sujets épineux (responsabilité du déracinement de plusieurs centaines de milliers de personnes, problèmes historiques liés à l'Empire ottoman, populations minoritaires dans les deux pays, etc.), il devient plus difficile de publier les ouvrages tels qu'ils sont: soit les ouvrages sont saisis, soit dans les traductions, une certaine autocensure est pratiquée. Selon R. Zarakolu, dans les années 50, la traduction de l'œuvre d'Ilias Vénézis fut interdite; en 1982, l'ouvrage de Dido Sotiriou, les Terres ensanglantées, publié dans les Éditions Alan, sous la direction de Ragip et Aye Zarakolu fut saisi par le régime militaire sous le prétexte qu'il " portait atteinte à la turcité et à l'armée turque $»^{43}$; ces deux éditeurs furent traînés devant les tribunaux plusieurs dizaines de fois pour les ouvrages qu'ils ont publiés ${ }^{44}$. Aye Nur Zarakolu fut arrêtée et emprisonnée durant dix mois aussi bien par le régime militaire de 1980 que par le régime civil. D'ailleurs, le titre de l'ouvrage de Dido Sotiriou a été changé délibérément pour être rebaptisé en turc : Dites bonjour de ma part à l'Anatolie. Il a fallu que le prix de la Fondation Abdi Ipekçi fût décerné en 1982 à ce livre pour qu'il puisse être diffusé. Herkül Millas qui s'est penché sur la question de l'autocensure dans les traductions des textes grecs en turc a remarqué que parmi plusieurs dizaines de romans, un seul a paru sans censure, et encore celui-ci a été déféré devant les tribunaux; que dans le roman de Dido Sotiriou, tous les passages qui étaient contre la Turquie ou les Turcs ont été supprimés. Selon $\mathrm{H}$. Millas, même les poèmes de Séféris ont paru censurés ${ }^{45}$. 
Mais dans ce domaine de la censure ou de l'autocensure, il faut donner quelques exemples afin d'illustrer à la fois la manière dont les traducteurs prennent des libertés avec les textes originaux, ou encore les maisons d'éditions, soit par contrainte de la justice, soit par souci de volontarisme pour rapprocher les Grecs et les Turcs, tolèrent (en tout cas toléraient jusqu'à une date récente) ce type d'amputation. Aussi dans une étude comparative, on relève que dans Loxandra de Maria Iordanidou plusieurs passages sont soit tronqués soit carrément supprimés ${ }^{46}$. Les expressions qui désignent les klzllbaş (Alevi) comme des fanatiques ou des damnés sont supprimées; de même, dans le texte original, on parle de massacres à Chios et à Adana, alors que dans la traduction, ces expressions sont atténuées pour être traduites comme les "soulèvements" ou des "crimes»; l'auteur fait dire à Loxandra pour les dirigeants turcs des mots comme " chiens ", " choléra " ou encore "le fouet de l'humanité », or en turc, ces mots ne sont pas traduits; mieux la phrase "nous n'avions aucune relation avec les chiens de musulmans» est traduite ainsi : "Nous n'avions aucune relation avec les sultans». Ainsi, l'éditeur et le traducteur versent dans l'idéologie kémaliste qui se distingue notamment par le dénigrement systématique de la période ottomane. Même l'expression comme «l'Ottoman en faillite économiquement » est tronquée pour être traduite "l'Ottoman en faillite ». On connaît la lecture quelque peu manichéenne de Kazantzakis dans les Lettres au Greco où l'auteur identifie Marie à la Grèce (le bien) et le diable à la Turquie (le mal). Or, dans la traduction en turc, ces oppositions sont gommées pour présenter au lecteur turc un texte plus " dépouillé ». Voici un tableau indiquant ces différences.

Tableau II : autocensure dans les Lettres au Greco

\begin{tabular}{|c|c|}
\hline $\begin{array}{l}\text { Texte original } \\
\text { Édition Athènes, } 1982\end{array}$ & $\begin{array}{l}\text { Traduction en turc } \\
\text { Par Ahmet Angın, Éditions E, Istanbul, } 1975\end{array}$ \\
\hline «À cause de la peur du Turc» (p. 61). & «À cause de la peur » (p. 60). \\
\hline «C'était le diable turc brun » (p. 69). & (n'a pas été traduit du tout, p. 69). \\
\hline $\begin{array}{l}\text { «Je passais très vite par le quartier des } \\
\text { Turcs, à cause des odeurs qu'ils } \\
\text { dégageaient » (p. } 71) \text {. }\end{array}$ & $\begin{array}{l}\text { « Mais un jour, je suis passé très vite par le quartier des } \\
\text { Turcs » (p. 71). }\end{array}$ \\
\hline $\begin{array}{l}\text { « Voilà, dis-je, il leva le drapeau et partit } \\
\text { à la guerre ; ceux qui sont à terre sont des } \\
\text { Turcs » (p. 74). }\end{array}$ & $\begin{array}{l}\text { « Voilà, dis-je, il leva le drapeau et partit à la guerre » } \\
\text { (p. 76). }\end{array}$ \\
\hline $\begin{array}{l}\text { «Moi, je me bats contre le Turc, je fais ce } \\
\text { que je dois faire, fais attention, que le } \\
\text { Franc ne te trompe pas» (p. 100). }\end{array}$ & $\begin{array}{l}\text { «Moi, je me bats, je fais ce que je dois faire, bats-toi } \\
\text { aussi, fais attention, que le Franc ne te trompe pas" } \\
(104) \text {. }\end{array}$ \\
\hline $\begin{array}{l}\text { «Tous mes aïeuls que les Turcs ont tués } \\
\text { pendant la guerre, toutes mes aïeules } \\
\text { persécutées et dont les seins arrachés par } \\
\text { les Turcs» (p. 110). }\end{array}$ & $\begin{array}{l}\text { "Tous mes aïeuls morts pendant la guerre, toutes mes } \\
\text { aïeules arrêtées et tombées de faiblesse » (p. 115). }\end{array}$ \\
\hline
\end{tabular}


\begin{tabular}{|l|l|}
\hline «Mon grand-père pensait aux Juifs & «Si mon grand-père était là, il penserait aux Juifs \\
crucificateurs et aux Turcs, puis il & $\begin{array}{l}\text { crucificateurs, il prendrait son bateau, se mettrait à } \\
\text { travers le Détroit pour se battre avec les navires } \\
\text { attaquait les navires turcs et nageait } \\
\text { ainsi »(p. 238). }\end{array}$ \\
$\begin{array}{l}\text { musulmans et ainsi en se vengeant, il se sentait léger » } \\
\text { (p. 271). }\end{array}$
\end{tabular} d'intégrer l'Union européenne interdit, en tout cas limite considérablement la censure. Il n'est pas insensé de penser que désormais la littérature grecque comme les autres littératures d'ailleurs, pourra être traduite en turc sans censure ni autocensure. De toute manière, les œuvres construites sur les différends gréco-turcs étant de plus en plus rares chez la dernière génération d'auteurs, les traductions seront faites non pas en fonction des thèmes qui y sont traités, mais en fonction de leur qualité. C'est le cas précisément de Kostas Mourselas.

\section{Pour conclusion : Mourselas, Millas et les autres}

En effet, le dernier auteur grec qui occupe les devantures des librairies en Turquie est Kostas Mourselas. Son livre Vammena Kokkina Malia ${ }^{47}$ (La Rousse aux cheveux teints/ Kızıla Boyalı Saçlar1 ${ }^{48}$ ) a attendu longtemps avant d'être publié en turc. En effet, son traducteur Kosta Saraoğlu a cherché pendant un certain temps, avant de trouver un éditeur. Finalement, le livre a été publié par les Éditions OM avec une subvention du Ministère de la Culture grecque. Mais le livre a atteint rapidement des records de vente, car en 2001, il fut parmi les livres les plus vendus en Turquie (30 éditions, 60000 exemplaires vendus, et près de 100000 exemplaires tirés illégalement) ${ }^{49}$. Réédité par İş Bankası Kültür Yayımları, le livre continue à susciter un certain intérêt et surtout à créer un certain engouement pour la littérature grecque en Turquie, au point que le Président de la République s'est fait filmer par les télévisions en achetant le livre dans une librairie. Du même coup, un second livre du même auteur : Nous sommes fermés pour cause de deuil, est traduit en turc et publié par la même maison d'édition. Le même auteur est venu en Turquie participer à la foire du Livre pour signer ses livres.

Si les choses vont de cette manière, la littérature grecque en Turquie connaîtra un essor grandissant. Un signe avant-coureur en est que pour la première fois neuf maisons d'éditions grecques ont participé à la foire du livre d'Istanbul, en 2003.

Kızıla Boyalı Saçlar ouvre probablement une période irréversible où, désormais, la littérature grecque est traduite directement à partir du grec, et non pas, comme on le faisait beaucoup à partir du français ou de l'anglais. Mais dans ce domaine de la promotion de la littérature néo-hellénique, le rôle d'un turco-grec est à souligner très fortement. Fondateur, en 1990, du département de la langue et la littérature grecques contemporaines à l'Université d'Ankara, Herkül Millas a traduit un grand nombre d'ouvrages en turc.

41 Ses travaux portant sur l'image respective dans les littératures grecque et turque sont des œuvres d'avant-garde sous des couleurs parfois satiriques. Et il est l'un de ceux qui tentent de faire connaître la littérature grecque en Turquie. À juste titre, il indique qu'en Turquie, l'on choisit les livres et écrits qui " plaisent » aux Turcs, pour montrer " comment les Grecs nous aiment », ou alors inversement, et pas forcément dans les 
mêmes milieux éditoriaux, l'on choisit les morceaux les plus haineux pour les utiliser comme matériels de propagande. Or, H. Millas part de l'hypothèse qu'il faut connaître "l'autre réel», et non pas l'autre imaginaire, afin de ne pas subir le choc de la déception. En conséquence, l'histoire, les croyances, les préjugés grecs sont à connaître avant toute entreprise. Il le fait par le biais de Vénézis, de Ritsos, de Cavafis, d'Élytis, de Séféris et bien d'autres, non pas comme un représentant de la littérature grecque, mais au moins comme un moyen de déceler les tendances, les commentaires et les perceptions de la littérature grecque inconnue en Turquie $e^{50}$.

Le rapprochement gréco-turc, la solution des grands dossiers qui divisent les deux pays comme le problème chypriote, la question égéenne et celle des minorités, le processus d'intégration amorcé de la Turquie dans l'Union européenne promettent un avenir meilleur à la littérature grecque en Turquie.

\section{NOTES}

1. Sous le titre de Kitab 'ussemaniye, cette traduction est due à Esat Efendi de Ioannina (décédé en 1730). Pour tout cela voir Vedat Günyol, «Türkiye Çeviri», Cumhuriyet Dönemi Türkiye Ansiklopedisi, vol. 2, İleşitim Yayınları,(s.l.s.d), pp. 324-325.

2. şerif Hulüsi, « Tercüme ve Dünya Edebiyatı », Tercüme, n 3, 19 septembre 1940, pp. 283-296.

3. «Varlık ne için çıkıyor?» (Pourquoi Varlık paraît-elle ?), Varlık, nº 1, 15 juillet 1933.

4. Première nouvelle publiée : «Akşam Karanlığı " (L'obscurité du soir) d'Étienne Tomorkény, traduite par Muzaffer Resit, Varlk, nº 1, 15 juillet 1933, p. 10.

5. Première nouvelle publiée : «Kurban» (La victime) de L. Nikolova, traduite par Ali Haydar, Varlik, n 7, 15 octobre 1933, p. 108.

6. À partir du numéro 82 ( $1^{\mathrm{er}}$ décembre 1936$)$, les auteurs des textes étrangers sont signalés en couverture.

7. Je remercie Sara Yontan, de la Bibliothèque nationale de France, d'avoir mis si aimablement à ma disposition la collection complète de la revue Varlı.

8. Naturellement, pour les « romans ", il s'agit des extraits.

9. Extrait de Sti sikamy apo kato.

10. Rédacteur en chef du journal Iho Tis Ellados.

11. Directeur du journal littéraire Néa Estia.

12. Avram Papazoglu, "Türkiye ile Yunanistan Arasmda Fikrî Münasebetler " (Les relations intellectuelles entre la Turquie et la Grèce), Varlı, vol. 7, $\mathrm{n}^{\circ} 144$ ( $1^{\mathrm{er}}$ juillet 1939), pp. 395-397.

13. Damla Damla fut la première l'œuvre publiée en turc moderne.

14. Ruşen Eşref Ünaydım fut le chroniqueur officieux du régime kémaliste tout en donnant certaines œuvres littéraires. Ainsi, le groupe littéraire Parnassos grec l'avait élu comme membre honoraire.

15. Avram A. Papazoğlu, Varlık, loc. cit, p. 396.

16. Le $1^{\text {er }}$ numéro de cette revue de théâtre a paru, le 15 février 1930. Elle avait pour objectif de diffuser le programme du théâtre "Darülbedâyi », mais aussi de donner des informations sur le théâtre mondial.

17. Tercüme, $\mathrm{n}^{\circ}$ 29-30, 19 mars 1945 , p. II. Le volume contient 486 pages. Le double numéro suivant ( $\mathrm{n}^{\circ} 31-32,19$ juillet 1945) est de la même manière consacré aux textes anciens grecs, mais aussi à 
la littérature occidentale classique : Becker, Nietzsche, Barrès, Alain, André Gide, Paul Valérie,

E. Renan, etc.

18. Ibid. p. III.

19. Cette étude est faite par Herkül Millas et Damla Demirozü. Je remercie les auteurs de me l'avoir communiquée.

20. Destansi Öykü, De Yayımları, Istanbul, 1965 ; Üç Kırmızı Güvercin, Altın Kitaplar Yayımları, Istanbul 1971 ; Profil, Yapı Kredi Yayımları, Istanbul, 1995.

21. Bütün Siirleri, Varlık Yayımları, Istanbul 1990.

22. La revue publiée par Türk Dil Kurumu, à partir de 1933 et qui continue à paraître.

23. Sous la direction de Hüsamettin Bozok, la revue commença à paraître à Istanbul, le $1^{\mathrm{er}}$ avril 1950.

24. Publiée par Muhtar Ebata et Vedat Günyol, cette revue d'opinion et de littérature est une suite de la revue Ufuklar (1952-1953) de Orhan Burian.

25. Revue publiée par les Éditions Varlık à partir de 1967.

26. Yazko Çeviri dit «avoir pour but de transmettre la pensée contemporaine mondiale par des traducteurs de talents ».

27. Varlık Yıllığı, 1982, Istanbul, Varlık Yayımları, pp. 31-32.

28. Varlık Yıllığı, 1984, Istanbul, Varlık Yayımları, p. 269.

29. Ibid. p. 270.

30. La traduction en turc était faite par Cevat Çapan. Yannis Ritsos, Bütün şiirlerinden Seçmeler, Kavram Yayımları, Istanbul, 1995, pp. 219-221. Nous avons fait cette traduction à partir du turc.

31. Cette dernière rencontre a eu lieu à Karlovassi en 1981.

32. Édité pour la première fois en Grèce en 1946.

33. Traduction d'Osman Bleda, Éditions Belge, collection Marenostrum, 1993. L'ouvrage est traduit en français par Jacques La Carrière sous le titre Chronique d'une cité, avec une introduction d'André Chamson, Gallimard, 1960.

34. P. Prévélakis, Chronique d'une cité, introduction en français, p. 14.

35. Ce prix fut créé par Andreas Politakis, un ingénieur grec, après l'assassinat du journaliste Abdi İpekçi qui a tant œuvré pour rapprocher les journalistes turcs et grecs.

36. Extraits du texte qui est placé au début ou à la fin de chaque ouvrage publié dans cette collection.

37. Pièces de monnaie frappées par le Sultan Abdülmecid (règne : 1839-1861).

38. Benden Selam Soyle Anadolu'ya, pp. 44-45.

39. Marenostrum, 2000.

40. Né en 1932, il fait partie de la génération des années 60.

41. Né à Mytilène vers 1890 ou 1892. A enseigné aux Langues Orientales.

42. Yorgos Andreadis, Tamama, Pontus'un Yitik Kızı, Belge Yayımları, $3^{\mathrm{e}}$ édition, Istanbul, 1981, p. 110.

43. Ragıp Zarakolu, Yeniden Ozgür Gündem, 12 nov. 2003.

44. Entretien par correspondance avec Ragıp Zarakolu, 21 février 2004.

45. Herkül Millas, Zaman, 27 janvier 2004.

46. Cette étude inédite est faite par Damla Demirözü et Herkül Millas à partir des textes originaux et des traductions. Je remercie également Ash Igsu de m'avoir communiqué ses travaux sous presse: "Cultural Resistance and Geographie Kinship in Framing Narratives of the TurkishGreek Population Exchange", in Esra Ozyürek (dir.), Politics of Public Memory in Turkey, Syracuse University Press, 2007.

47. Sa première édition en grec date de 1989 (Éditions Kédros).

48. Première édition, mars 2000, traduction faite par Kostas Sarioğlu que je remercie pour l'entretien qu'il m'a accordé et qui croit en l'avenir de la littérature grecque en Turquie.

49. Ce livre aurait vendu en Grèce 260000 exemplaires. 
50. H. Millas, Ayvalik ve Venezis, Yunan Edebiyatinda Türk Imaji, Istanbul, 1998, pp. 9-10.

\section{RÉSUMÉS}

Jusqu'à la fin de la Première Guerre mondiale, mis à part quelques classiques, la littérature grecque n'a pas bénéficié des efforts de traduction des pouvoirs publics ottomans. C'est donc à la période républicaine que l'on doit une volonté politique et idéologique pour se servir de la littérature grecque comme moyen de rapprocher les deux peuples, turc et grec. La revue Varlik a joué dans ce domaine le rôle pionnier à partir de 1933. Mais c'est au ministre de l'Éducation nationale Hasan Ali Yücel (1897-1961) que la Turquie doit la véritable révolution de traduction. Fervent défenseur de la philosophie et de la littérature classiques grecques, Yücel a fait traduire pratiquement tous les textes principaux de la période hellénistique, mais a fait publier également la revue Tercüme où de nombreux auteurs grecs ont été présentés au lecteur turc, avant que les grands poètes contemporains comme Cavafis, Séféris, Kazantzakis, Élytis, Ritsos et d'autres encore soient abondamment traduits à partir des années 60. La collection Marenostrum à partir de 1977 a fait œuvre militante afin de faire connaître la littérature grecque des années 20 et 30. Selon toute vraisemblance, aujourd'hui la littérature grecque en Turquie n'a plus besoin de «prouver» autre chose que sa valeur littéraire: une nouvelle génération d'écrivains, comme Mourselas est en train de voir le jour.

Until the end of World War 1 and except for a some classics, Greek literature did not enjoy the ottoman authorities efforts of translation. The translation movement is due to the Republican political and ideological will to use Greek literature as a means of bringing closer the two peoples, the Turkish and the Greek one. From 1933 the Varlik review has acted as a pioneer in this field, but Turkey owes his true revolution in translation to the minister of Education Hasan Ali Yücel (1897-1961). As an ardent defender of classic Greek literature and philosophy, Yücel has not only had almost all the main texts from the Hellenistic period translated but has also had the Tercüme Dergisi (Review Translation) brought out where many Greek authors have been introduced to Turkish readers before great contemporary poets such as Kavafis, Seferis, Kazantzakis, Elytis, Ritsos and many more world known beeing prolifically translated from the 60 's. From 1977, the Marenostrum collection campaigned for the Greek literature of the 20's and 30 's to be known. In all possibility today Greek literature doesn't need any longer to "prouve" anything else than its literary value: Mourselas, is coming into being.

Birinci Dünya Savaşı'nın sonlarına kadar, birkaç klasik dışında, Yunan Edebiyatı Osmanlı Kamu Güçleri'nin çeviri çabalarından yararlanmamıştır. Daha çok Cumhuriyet Dönemi'nde Yunan Edebiyatı'nın, siyasal ve ideolojik nedenlere bağlı olarak, Türk ve Yunan halkını birbirine yakınlaştırmak amacıyla kullanıldığını görüyoruz. Varlık Dergisi bu alanda 1933'ten itibaren öncü bir rol oynamıştır. Ancak Türkiye çeviride asıl devrimi Milli Eğitim Bakanı Hasan Ali Yücel (1897-1961) döneminde yapmıştır. Klasik Yunan Felsefesi ve Edebiyatı'nın ateşli savunucusu Yücel Helenistik Dönem'e ait fiilen bütün ana metinleri çevirtmiş, aynı zamanda bunları birçok Yunan yazarının Türk okuruna sunulduğu Tercüme Dergisi'nde yayınlatmış, Kavafis, Seferis, Kazancakis, Elitis, Ritsos gibi büyük çağdaş şairleri ve daha birçokları 60'lı yıllardan itibaren büyük ölçüde çevrilmiştir. Marenostrum Koleksiyonu 1977'den itibaren, 20'li ve 30'lu yılların Yunan Edebiyatı'nı tanıtmak için mücadele etmiştir. Görünüşe göre Türkiye'de artık Yunan 
Edebiyatı'nın edebi değerini ispat etmenin dışında başka bir şeye ihtiyacı yok: Mourselas gibi, yeni bir nesil yazar yetişmekte.

\section{INDEX}

Index géographique : Grèce, Turquie motsclestr Elytis Odysseas (1911-1996), Iordanidou Maria (1897-1989), Kavafis Constantin (1863-1933), Kazancakis Nikos (1883-1957), Myrivilis Stratis (1890-1969), pontus, Prevelakis Pandelis (1909-1986), Ritsos Yannis (1909-1990), Seferis Yorgo (1900-1971), Sotiryou Dido (1909-2004), tercûme, turk edebiyati, Venezis Ilyas (1904-1973), yunanli edebiyati

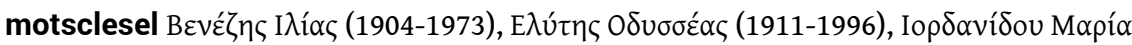

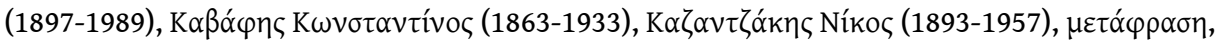

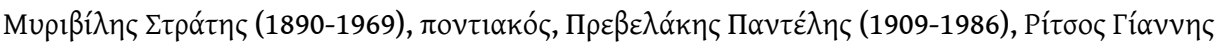

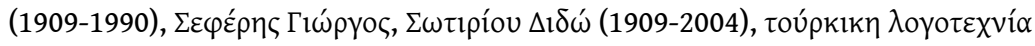
Thèmes : Littérature, Sciences politiques Mots-clés : Cavafis Constantin (1863-1933), Cavafis Constantin (1863-1933), Elytis Odysseas (1911-1996), Elytis Odysseas (1911-1996), Iordanidou Maria (1897-1989), Iordanidou Maria (1897-1989), Kazantzakis Nikos (1883-1957), Kazantzakis Nikos (1883-1957), Myrivilis Stratis (1890-1969), littérature grecque, Prévélakis Pandelis (1909-1986), littérature turque, Myrivilis Stratis (1890-1969), Ritsos Yannis (1909-1990), Séféris Giorgos (1900-1971), pontique, Prévélakis Pandelis (1909-1986), Sotiriou Dido (1909-2004), Ritsos Yannis (1909-1990), Vénézis Ilias (1904-1973), Séféris Giorgos (1900-1971), Sotiriou Dido (1909-2004), Kizılbaş, traduction, Mourselas Kostas (1932-), Vénézis Ilias (1904-1973), Özdemir Ince (1936-), Papadopoulos Giorgos (1919-1999), Pont/Pontique, Ponticité, Samarakis Antonis (1919-2003), Türk Dil Kurumu, Metaxás Ioannis (1871-1941)

motsclesmk ГРЧКИ ЛИТЕРАТУРА, ПРЕВЕДУВАњЕ, ТУРСКИ ЛИТЕРАТУРА

Index chronologique : vingtième siècle

Keywords : Greece, Turkey, Pontus, twentieth century, Myrivilis Stratis (1890-1969), Greek literature, literature, translation, Ritsos Yannis (1909-1990), politics, Turkish literature, Seferis Giorgos (1900-1971), Kavafis Constantin (1863-1933), Kazantzakis Nikos (1883-1957), Elytis Odysseas (1911-1996), Venezis Ilias (1904-1973), Prevelakis Pandelis (1909-1986), Sotiriou Dido (1909-2004), Iordanidou Maria (1897-1989) 